\title{
Completion pneumonectomy after fenestration for empyema due to nontuberculous mycobacteriosis associated with destroyed lung as a result of cancer surgery
}

\author{
Ryo Takahashi $^{1,2,3}$, Taiki Fujiwara ${ }^{1,2}$, Hisami Yamakawa ${ }^{1,2}$ \\ ${ }^{1}$ Department of General Thoracic Surgery, National Hospital Organization Chiba-East Hospital, Chiba, Japan; ${ }^{2}$ Department of General Thoracic \\ Surgery, Graduate School of Medicine, Chiba University, Chiba, Japan; ${ }^{3}$ Department of Surgery, Jinken Clinic, Kanagawa, Japan \\ Correspondence to: Ryo Takahashi, MD, PhD. Department of General Thoracic Surgery, National Hospital Organization Chiba-East Hospital, \\ 673 Nitona-cho, Chuo-ku, Chiba, Japan. Email: blackbelt2000g@nifty.com.
}

\begin{abstract}
Long-term follow-up of post-operative lung cancer patients indicates that some patients develop lung complications. Destroyed lung cannot be ignored because it predisposes the patient to recurrent infection. We report a case of thoracic empyema with bronchopleural fistula that developed in lung tissue damaged due to cancer surgery and associated with an infection of Mycobacterium gordonae (M. gordonae); a class of bacterium responsible for nontuberculous mycobacterial infection. The patient's cancer did not recur after surgery and followed a typical course that began with sub-pleural cystitis followed by repeated infection. We performed fenestration because the patient developed fistulous empyema and the infection became difficult to control using antibiotics. The patient then underwent a radical pleuropneumonectomy and his postoperative course was excellent. Thus, in cases of impaired lung function due to cancer surgery and where pharmacologic control of infection is challenging, we suggest that radical surgical intervention should be considered.
\end{abstract}

Keywords: Completion pneumonectomy; fenestration; destroyed lung; nontuberculous mycobacteriosis; Mycobacterium gordonae (M. gordonae)

Submitted Apr 26, 2017. Accepted for publication Oct 10, 2017.

doi: $10.21037 /$ jtd.2017.10.72

View this article at: http://dx.doi.org/10.21037/jtd.2017.10.72

\section{Introduction}

Mycobacterium gordonae (M. gordonae) belongs to genus II in the Runyon classification system and its pathogenicity is thought to be extremely low. However, in recent years it has attracted increasing attention since it has been reported to cause the nontuberculous mycobacterial infections often observed in individuals with human immunodeficiency virus (HIV) infection (1). In addition, the incidence of nontuberculous mycobacterial infection is increasing worldwide (2-4). Although the Mycobacterium avium complex (MAC) is known to account for the majority of nontuberculous mycobacterial infections, the range of causative bacteria has now become diverse. Among these, $M$. gordonae has been successfully treated with rifampicin (RFP), ethambutol (EB), and clarithromycin (CAM). However, medical management is often difficult because these drugs do not completely eliminate the bacteria. Although it has been suggested previously that surgical intervention is necessary in patients who develop hemoptysis (5), there is no consensus on which specific surgical method is recommended. Here we report a case of a patient with destroyed lung as a result of surgery for lung cancer. The patient presented with repeated hemosputum and developed fistulous empyema which was treated successfully with fenestration and pleuropneumonectomy. In this present case the patient had been previously managed using surgery with one course of post-surgical intrathoracic chemotherapy, but had not received radiotherapy. 

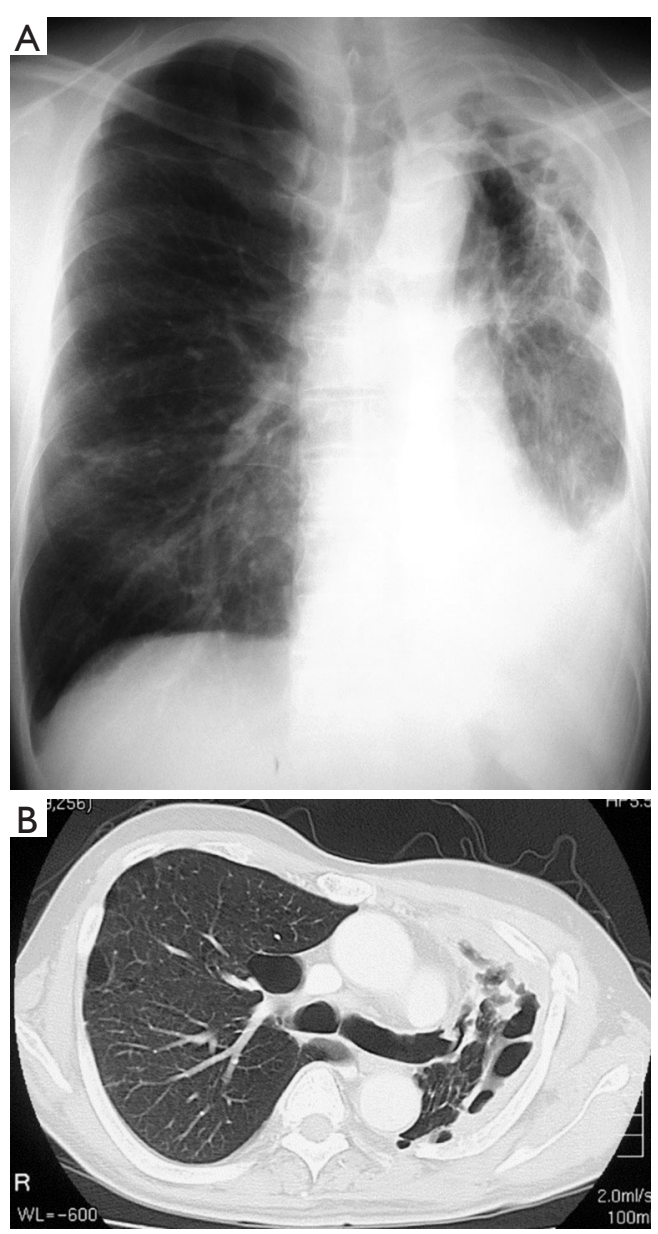

Figure 1 Chest X-ray (A) and chest CT (B). Multiple cysts were observed directly under the pleura causing narrowing of the lung and were associated with the severity of lung damage. The structure of the lung had been almost completely destroyed by repeated infections.

\section{Case presentation}

Case: a 59-year-old man.

Past history: the patient underwent left upper lobectomy with lymph node dissection for left lung cancer at 45 years of age.

Pathological findings: large cell carcinoma, pT2N0M0

Clinical course: post lung cancer surgery, no adjuvant therapy was added. Chest CT revealed cyst formation in the apical area at the surgical site approximately 3 years post-surgery. The patient developed hemosputum around 4 years after surgery and a yellow sputum 5 years after surgery. A sputum smear test revealed a nontuberculous mycobacterial infection which was treated with RFP, EB, and CAM. Bacteria were not detected in sputum or in blood
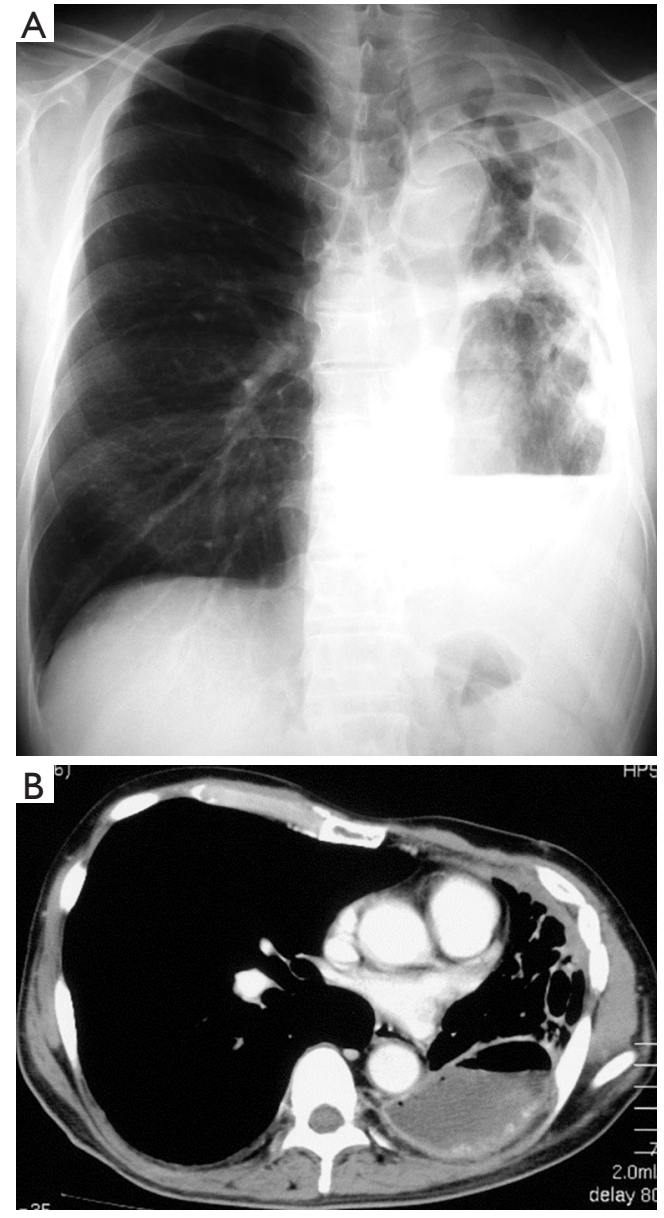

Figure 2 Chest X-ray (A) and chest CT (B). There was an apparent air-fluid interface visible on images, leading to the diagnosis of fistulous empyema.

culture. After 1 year of chemotherapy, the production of hemosputum and yellow sputum was reduced. Seven years after surgery the patient developed fever and recurrence of hemosputum production and consequently received the same antibiotic regimen for a further year. Nine years after surgery, a chest CT revealed changes to the destroyed lung. Ten years after surgery a sputum culture once again revealed a $M$. gordonae infection (Figure $1 A, B$ ). Eleven years after surgery a chest CT revealed the presence of fluid and a fistulous empyema (Figure $2 A, B$ ). The patient subsequently developed pneumonia in the contralateral lung after aspiration of the empyema from the ipsilateral lung. We discussed whether to continue the conservative therapy and whether to perform a surgery at the joint conference between departments of internal medicine and 


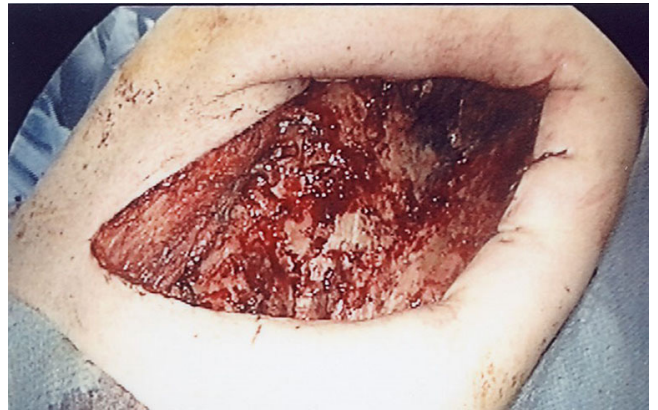

Figure 3 Operative findings of fenestration. We curetted the necrotic material and ferruginous liquid component in the thoracic cavity.

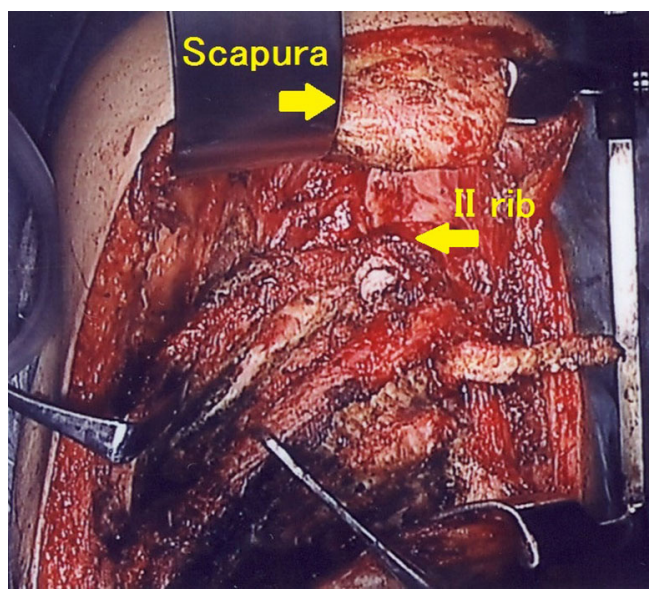

Figure 4 Operative findings of pleuropneumonectomy. A $1 \mathrm{~cm}$ thick callous that extended over the entire surface of the lung was observed. The phrenic nerve, vagus nerve, and sympathetic trunk were all rigidly fixed to this pleural callous.

surgery. Since the disease progression became difficult to control by pharmacotherapy, we decided to perform a surgery and fenestration was performed (Figure 3). We curetted the necrotic material and ferruginous liquid in the thoracic cavity. Inflammation was improved drastically by fenestration. The patient's physical strength returned after dietary intervention allowing them to undergo a radical pleuropneumonectomy (Figure 4). Their postoperative course was uneventful and the patient is currently under follow-up in an outpatient's clinic.

\section{Discussion}

Nontuberculous mycobacterial infection is environmentally contracted since the bacterium responsible is present in soil, dust, water and food. The number of known acid-fast bacilli variants has increased from year to year and there are currently more than 100 types of which more than 20 are reported in association with infections in humans (5). Previously, the pathogenicity of these bacteria in humans was thought to be extremely low, but this type of infection has attracted more attention recently due to the increased detection of $M$. gordonae infection in HIV-positive individuals. Since the first report of pulmonary $M$. gordonae infection by Ohta et al. in 1987 (6), there have been several reports of such cases in Japan. In these cases, images of the chest often show progression of characteristic patterns that can include cavity formation, worsening bronchodilatation, and enlargement of nodular shadows. It has also been suggested that disease may result if antibiotic treatment is not started quickly $(7,8)$.

There are no standard treatment guidelines for $M$. gordonae infection which is a relatively rare form of bacterial infection. It has been commonly treated with multiple drugs including isoniazid (INH), REF, EB, and CAM $(7,8)$. The effectiveness of these drugs has been demonstrated by discontinuation of discharge, general improvement in symptoms and the reduction in the size of nodular shadows. However, long-term treatment outcomes have not yet been reported. According to the American Thoracic Society (ATS), surgery is indicated if the response to antibiotics is poor or the patient develops complications such as hemoptysis. However, there are also not yet any long term reports on surgical outcomes (5).

M. gordonae infection is an opportunistic infection associated with lowered immunity. In the present case, the patient had destroyed lung as a result of surgery for lung cancer and presented with repeated hemosputum and fever. The sputum culture led to the diagnosis of nontuberculous mycobacterial infection and infection with $M$. gordonae was subsequently confirmed. The patient's history of destroyed lung, combined with evidence gleaned from similar infections in HIV patients, suggests there is a relationship between a weakened host and the consequent, relatively high, infectivity of $M$. gordonae. In fact, antibiotic therapy with RFP, EB and CAM produced a decreased bacterial load, but chest images revealed continued deterioration. Thus the infection became difficult to control using conventional antibiotics and lead to the development of empyema in association with pneumonia in the contralateral lung after aspiration of the contents of the empyema. Inflammation was dramatically improved by fenestration, 
and radical pleuropneumonectomy was performed in the residual lung to treat the empyema.

Radical surgery for chronic thoracic empyema includes decortication (9), thoracoplasty, and pleuropneumonectomy. Although mortality after pleuropneumonectomy for chronic thoracic empyema is reported to be $5.4 \%$ to $8.5 \%$ $(10,11)$, the incidence of postoperative empyema, a major complication, is now as low as $9.7 \%$ to $45.7 \%(10,11)$. In cases of fistulous empyema that develop as a result of destroyed lung, pleuropneumonectomy is indicated due to the risk of pneumonia in the contralateral lung after surgical intervention $(10,11)$. However, because there is a high risk of complications, this procedure is not universally recommended (12) and its indication should be carefully considered $(11,13)$. Millar $e t a l$. pointed out that risk factors for surgical death include old age, preoperative use of steroids, and low preoperative hemoglobin levels (14). Chataigner et al. reported that renal failure is a risk factor responsible for surgical death (15). Concerning indications for extrapleural pneumonectomy which involves the remaining lung and pleura, a unified evaluation relating to cardiopulmonary function has not yet been established. Ono et al. reported that prediction of postoperative capacity of the remaining lung $\left(800 \mathrm{~mL} / \mathrm{m}^{2}\right.$ or more and FEV1.0 $\left.\geq 600 \mathrm{~mL} / \mathrm{m}^{2}\right)$ is one of the surgical indications (16). However, prediction of postoperative lung function is just one of the indicators; therefore, it is important to determine surgical indications after comprehensive evaluation of patients' general conditions, including their performance status (PS).

In our present case, because drug therapy for inflammation was not effective, drainage of the thoracic cavity was achieved by fenestration, and the chronic inflammation drastically improved. After nutritional supplementation the patient achieved the strength required for surgery and underwent a radical pleuropneumonectomy of the residual lung to treat the empyema. The postoperative course was uneventful and the patient is currently under follow-up at an outpatients' clinic. Thus, when treating nontuberculous mycobacterial infections for which antibiotic therapy has proved ineffective, radical surgical treatment should be considered.

\section{Conclusions}

We report a case of fistulous empyema in association with $M$. gordonae infection (one of the bacteria responsible for nontuberculous mycobacterial infections), in destroyed lung as a result of cancer surgery, that was successfully treated with fenestration and pleuropneumonectomy. We suggest it is necessary to carefully consider the indications for this procedure on a case by case basis i.e., a full preoperative assessment should be performed, including confirmation of the nature of the bacterial infection and an assessment of the patient's competency before performing such a major surgical intervention.

\section{Acknowledgements}

None.

\section{Footnote}

Conflicts of Interest: The authors have no conflicts of interest to declare.

Informed Consent: Written informed consent was obtained from the patient for publication of this manuscript and any accompanying images.

\section{References}

1. Lessnau KD, Milanese S, Talavera W. Mycobacterium gordonae: a treatable disease in HIV-positive patients. Chest 1993;104:1779-85.

2. Bodle EE, Cunningham JA, Della-Latta $\mathrm{P}$, et al. Epidemiology of nontuberculous mycobacteria in patients without HIV infection, New York City. Emerg Infect Dis 2008;14:390-6.

3. Henry MT, Inamdar L, O'Riordain D, et al. Nontuberculous mycobacteria in non-HIV patients: epidemiology, treatment and response. Eur Respir J 2004;23:741-6.

4. Kennedy MP, O'Connor TM, Ryan C, et al. Nontuberculous mycobacteria: incidence in Southwest Ireland from 1987 to 2000. Respir Med 2003;97:257-63.

5. Griffith DE, Aksamit T, Brown-Elliott BA, et al. An official ATS/IDSA statement: diagnosis, treatment, and prevention of nontuberculous mycobacterial diseases. Am J Respir Crit Care Med 2007;175:367-416.

6. Ohta H, Sakashita T, Shiraishi T, et al. A case of pulmonary infection caused by M. gordonae. Kokyu (in Japanese) 1987;6:545-8.

7. Yanagisawa N, Miyamoto D, Ichinose $\mathrm{Y}$, et al. Pulmonary infection caused by Mycobacterium gordonae.

Kansenshogaku Zasshi 1999;73:482-5.

8. Fujita Y, Matsumoto H, Fujikane T, et al. Pulmonary infection caused by Mycobacterium gordonae in 
immunocompetent patient. Kekkaku 2000;75:369-74.

9. Massard G, Rougé C, Wihlm JM, et al. Decortication is a valuable option for late empyema after collapse therapy. Ann Thorac Surg 1995;60:888-95.

10. Odell JA, Henderson BJ. Pneumonectomy through an empyema. J Thorac Cardiovasc Surg 1985;89:423-7.

11. Shiraishi Y, Nakajima Y, Koyama A, et al. Morbidity and mortality after 94 extrapleural pneumonectomies for empyema. Ann Thorac Surg 2000;70:1202-6; discussion 1206-7.

12. Massard G, Dabbagh A, Wihlm JM, et al. Pneumonectomy for chronic infection is a high-risk procedure. Ann Thorac Surg 1996;62:1033-7.

13. Mouroux J, Maalouf J, Padovani B, et al. Surgical

Cite this article as: Takahashi R, Fujiwara T, Yamakawa H. Completion pneumonectomy after fenestration for empyema due to nontuberculous mycobacteriosis associated with destroyed lung as a result of cancer surgery. J Thorac Dis 2017;9(11):E997-E1001. doi: 10.21037/jtd.2017.10.72 management of pleuropulmonary tuberculosis. J Thorac Cardiovasc Surg 1996;111:662-70.

14. Miller DL, Deschamps C, Jenkins GD, et al. Completion pneumonectomy: factors affecting operative mortality and cardiopulmonary morbidity. Ann Thorac Surg 2002;74:876-83; discussion 883-4.

15. Chataigner O, Fadel E, Yildizeli B, et al. Factors affecting early and long-term outcomes after completion pneumonectomy. Eur J Cardiothorac Surg 2008;33:837-43.

16. Ono K, Nagashima A, Inaba G, et al. Completion Pneumonectomy for Postoperative Recurrent or Second Primary Lung Cancer. Jpn J Lung Canc 2009;49:157-61. 